detect MG and macrolide resistance by targeting the 23S rRNA gene. Nested PCRs were used to detect mutations in quinolone resistant determination regions in $g y r A, \operatorname{gyr} B$, parC and parE genes.

Results MG infection was detected in 27 MSM (17.1\%); 18 $(11.4 \%)$ at the genital site and $9(5.7 \%)$ at the rectal site. The bacterial load ranged from 2-32,700 genome copies/ $\mu$ l. Macrolide resistant MG was detected in 19 men (70.4\%), featuring typical 23S rRNA mutations (A2071G or A2072G transitions). One subject with MG had novel gene mutations (G1972T and G2038T) with unknown function. Eight (29.6\%) had fluoroquinolone-resistant $\mathrm{MG}$ harbouring parC mutations that cause changes in amino acid position 83 (S83I or S83R); 4 of them had an additional P62S mutation in parC and 1 had a F475S mutation in gyrB. Five men (18.5\%) had MG with dual macrolide and fluoroquinolone resistance. The prevalence of resistance was similar at rectal and genital sites.

Conclusion This is the first U.S. study to document a high frequency of macrolide and fluoroquinolone-resistant $\mathrm{MG}$ in HIV-infected MSM at rectal and genital sites. If these resistance mutations are associated with clinical treatment failure, more effective options to treat MG are needed.

\section{LB3.261 TRENDS IN CONDOM USE AND SEXUAL POSITIONING AMONG MEN-WHO-HAVE-SEX-WITH-MEN IN THE ERA OF HIV PRE-EXPOSURE PROPHYLAXIS, AND RISK FOR DIAGNOSES OF INCIDENT HIV AND OTHER SEXUALLY TRANSMITTED INFECTIONS - NEW YORK CITY, 2011- 2015}

Preeti Pathela, Kelly Jamison, Sarah Braunstein, Julia Schillinger. New York City Department of Health And Mental Hygiene, Queens, USA

\subsection{6/sextrans-2017-053264.496}

Introduction Men-who-have- sex-with-men (MSM) may modify sexual practices to reduce HIV and sexually transmitted infection (STI) risk. HIV pre-exposure prophylaxis (PrEP) may impact risk behaviour and STI acquisition.

Methods We matched HIV-negative MSM attending New York City (NYC) sexual health clinics during 2011-2015 to the NYC HIV registry in $01 / 2017$. We used visit-level data to assess trends in condom use during anal sex (consistent, inconsistent, no use; referent period $=3$ months), overall and by sexual positioning behaviour. We examined associations between condomless (inconsistent/no use) insertive, condomless receptive, and condomless versatile sex and incident HIV or STI (chlamydia/gonorrhoea/early syphilis). We used regression with generalised estimating equations (referent group $=$ anal sex with condoms), controlling for demographics, partner number, and STI history.

Results The proportion of visits with reported consistent condom use decreased from 2011-2015 (39\% to $31 \% \%$, $\mathrm{p}<0.001)$; inconsistent use increased $(48 \%$ to $55 \% \%)$, and no condom use was stable (13\%-14\%). There were significant declines in consistent condom use across all positioning categories. From all visits, MSM reported positioning as: 19\% condomless insertive, 9\% condomless receptive, 37\% condomless versatile, 35\% sex with condoms. For 25,216 STI testing visits that yielded 7438 diagnoses, all condomless-positioning categories were associated with incident STI; highest risk was with condomless insertive sex (aOR 1.8, 95\% CI 1.6-1.9). For MSM tested for HIV at 9744 visits, condomless receptive (aOR 2.8; 95\% CI 1.9-4.1) and condomless versatile sex (aOR 2.2; 95\% CI 1.6-2.8) were associated with incident HIV. Black MSM ( $25 \%$ of MSM) had the highest risk for STI and HIV (41\% of 368 new HIV diagnoses).

Conclusion We documented increases in condomless sex among a sentinel high risk group prior to and during the PrEP era. Insertive sex, perhaps perceived as a safer strategy, was associated with substantial STI risk when condoms were not consistently used. Assuring PrEP access for black MSM is critical.

\section{LB3.262 LATE POSTNATAL HIV MOTHER TO CHILD TRANSMISSION THROUGH BREASTFEEDING: ANALYSIS OF INFANT CASES OF PREVIOUSLY SERONEGATIVE MOTHERS INFECTED DURING LACTATION}

Ana Carolina Barcellos, Nathalia Zorze Rossetto, Cristina de Oliveira Rodrigues. Universidade Federal do Paraná, Curitiba - PR, Brazil

\subsection{6/sextrans-2017-053264.497}

Introduction Among children, the main postnatal HIV category of exposure is breastfeeding. When acute maternal infection occurs during lactation, the high maternal viral rate results in a higher risk of infant infection. There are few researches showing the impact of HIV infection on children of previously seronegative mothers, who acquire the HIV virus during breastfeeding. To elucidate the importance of this category of exposure, this study aims to evaluate HIV infection in infants assisted by a Paediatric Infectology Service in Brazil, emphasising the cases where vertical transmission occurred lately through breastfeeding.

Methods Transversal, analytical and descriptive study, with quantitative and qualitative approach analysing HIV infected patients from 0 to 16 years old. Data was collected during 2016, from charts of children assisted between 2010 and 2015. After previous selection, breastfed children of mothers who had negative HIV testing during pregnancy and/or at birth, had charts analysed in detail.

Results From a total of 122 cases, 95\% were mother-to-childtransmissions. Between these cases, 11 were considered possible/confirmed late postnatal transmission through breastfeeding. This group characterisation showed that at diagnosis, $72,7 \%$ presented significant and/or recurrent symptoms of HIV infection. In $45,4 \%$, mother and children were diagnosed at the same time and $72,7 \%$ of mothers were sexually infected.

Conclusion There was a significant prevalence of late postnatal transmission through breastfeeding in our sample. Differently from resource limited settings, most countries avoid breastfeeding by HIV infected mothers. Considering cases where women were infected only during lactation, our study highlights a gap in prevention of vertical transmission of HIV. The severity of infant symptoms, the moment of diagnosis and mother's category of exposure confirm the importance of preventive measures and scientific improvement to reduce postnatal HIV transmission. 


\section{Behavioural And Social Science Research}

\section{P4.01 ASSESSMENT OF COMPREHENSIVE HIVIAIDS KNOWLEDGE LEVEL AMONG IN-SCHOOL \& COMMUNITY LEVEL ADOLESCENTS OF NOAKHALI AND LAKSHMIPUR DISTRICT, BANGLADESH}

Abdur Rashid. Marie Stopes and Bapsa Consortium Project, Noakhali and Lakshmipur, Bangladesh

\subsection{6/sextrans-2017-053264.498}

Introduction In Noakhali and Lakshmipur, more adolescents are in school and community today than ever before; however, there are no studies that have assessed their comprehensive knowledge of HIV/AIDS. Thus, this study tried to assess the level of this knowledge and the factors associated with it among in-school and community level adolescents in Noakhali and Lakshmipur district.

Methods A cross-sectional school and community- based study was conducted using a facilitator-guided self-administered questionnaire conducted April 2016. The respondents were students attending regular school in 14 high schools and 70 community level adolescents located in 14 different Sub-districts in Noakhali and Lakshmipur district. The proportion of adolescents with comprehensive HIV/AIDS knowledge was computed and compared by sex. The investigating tool used was a prepared, pretested questionnaire. This had both openand close-ended questions related to various aspects of HIV/ AIDS. Different factors related to HIV/AIDS and information was gathered. The subjects were assessed for knowledge of various aspects of HIV/AIDS and associated risk factors. It was subsequently followed by an open discussion on HIV/ AIDS with the students. The factors that were associated with the comprehensive HIV/AIDS knowledge were assessed using multivariable logistic regression.

Results Only about one in four, 677 (24.5\%), in-school adolescents and community level adolescents have comprehensive HIV/AIDS knowledge. The knowledge was better among inschool adolescents from families with a relatively middle or high wealth index (adjusted OR [95\% CI] $=1.39$ [1.03-1.87] and 1.75 [1.24-2.48], respectively), who got HIV/AIDS information mainly from friends or mass media (adjusted OR $[95 \% \mathrm{CI}]=1.63[1.17-2.27]$ and 1.55 [1.14-2.11], respectively) and who received education on HIV/AIDS and sexual matters at school (adjusted OR $[95 \% \mathrm{CI}]=1.59 \quad[1.22-2.08]$ ). The females were less likely to have comprehensive HIV/AIDS knowledge compared to males (adjusted OR and [95\% CI] $=0.60[0.49-0.75])$.

Conclusion In general, only about a quarter of in-school adolescents had comprehensive HIV/AIDS knowledge. Although the female adolescents are highly vulnerable to HIV infection and its effects, they were by far less likely to have comprehensive HIV/AIDS knowledge. HIV/AIDS information, education and communication activities need to be intensified in high schools and rural community.

\section{P4.02 EFFECTS OF A RESTRICTIVE STATE LAW ON STD/HIV RATES IN OHIO}

${ }^{1}$ Abigail Norris Turner, ${ }^{1}$ Courtney N Maierhofer, ${ }^{1}$ Maria F Gallo, ${ }^{2}$ Carolette Norwood, ${ }^{2}$ Danielle A Bessett, ${ }^{1}$ Alison H Norris. 'Ohio State University, Columbus, USA; ${ }^{2}$ University of Cincinnati, Cincinnati, USA

\subsection{6/sextrans-2017-053264.499}

Introduction In recent years, Ohio has enacted multiple laws that impact sexual and reproductive health. In February 2016, a new law came into effect, which prohibits the state from contracting health services with any organisation that performs or promotes abortion. This law blocks funding of organisations such as Planned Parenthood from receiving state funds for activities related to HIV and STD testing and sexual education.

Methods We are conducting a rigorous evaluation of the sexual and reproductive health-related consequences of the new law. In an ecologic analysis of state- and county-level data from Ohio for 2015 and 2016, we will compare trends in prevalence of HIV, chlamydia and gonorrhoea (all nationally notifiable conditions) before and after the implementation of the2016 law. We will examine trends by several characteristics, including sex, race/ethnicity, age, education level, insurance status (Ohio is a Medicaid-expansion state under the Affordable Care Act), sexual orientation, urban/rural residence, and other variables. To disentangle the effect of the law from secular trends in HIV/STD, we will compare Ohio's outcomes to the same outcomes in nearby Illinois. Illinois has similar population characteristics to Ohio but does not have the same legislative environment impacting allocation of funds for reproductive health services.

Results Results will be available in summer 2017. In Ohio, we anticipate a decline in the number of HIV and STD tests performed in 2016 compared to 2015, and an increase in the prevalence of each disease in 2016 compared to 2015 . We anticipate no meaningful changes in trends across years in Illinois.

Conclusion Multiple states across the United States are considering legislation similar to Ohio's, to restrict the use of state and federal funding by clinical and community organisations that provide HIV/STD care alongside other sexual health services. Determining the HIV/STD-related impacts of such laws is critical to avoid putting men and women at higher risk of disease.

\section{P4.03 OPTIMISING DEVELOPMENT, IMPLEMENTATION AND EVALUATION OF STI/HIV PREVENTION INTERVENTIONS IN SUB-SAHARAN AFRICAN SCHOOLS: A QUALITATIVE STUDY WITH RESEARCHERS}

${ }^{1}$ Abubakar Sadiq Sani, ${ }^{1}$ Charles Abraham, ${ }^{1}$ Denford Sarah, ${ }^{2}$ Catherine Mathews. 'University of Exeter, Exeter, UK; ${ }^{2}$ South African Medical Research Council - Cape Town, South African Republic

\subsection{6/sextrans-2017-053264.500}

Introduction Existing trials show limited effectiveness of school-based sexual health interventions (SBSHI) in preventing sexually transmitted infections (STI) including HIV in subSaharan Africa (sSA). Limitations in content, implementation or evaluation are some reasons for this limited effectiveness. We explored facilitators and challenges to designing, implementing and evaluating SBSHI in sSA. 